\title{
Unusual presentation and treatment of isolated spontaneous gastric artery dissection
}

\author{
Ji Yeon Lim, Yoon Hee Choi, Sun Hwa Lee \\ Department of Emergency Medicine, Ewha Womans University School of Medicine, Seoul, Korea
}

Epigastric pain is a common symptom in the emergency department. Most epigastric pain is not fatal, except in acute myocardial infarction, aortic dissection, and abdominal aneurysm rupture. Epigastric pain can also be the only symptom of isolated spontaneous gastric artery dissection. We report the case of a 51-year-old woman diagnosed with neurofibromatosis who initially presented with only epigastric pain. She was initially misdiagnosed with gastritis, treated conservatively, and discharged. Two hours later, she returned to the emergency department with persistent epigastric pain and rebound tenderness in the epigastric area. Angiography revealed a left hepatic arterial aneurysm and right gastric artery dissection, which were subsequently embolized. Although extremely rare, patients presenting with epigastric pain at risk of vasculopathies should be considered for isolated spontaneous gastric artery dissection. Early recognition and definitive therapy lead to a favorable outcome.

Keywords Aneurysm; Dissection; Hepatic artery
elSSN: 2383-4625

Received: 25 March 2016

Revised: 16 April 2016

Accepted: 19 April 2016

Correspondence to: Yoon Hee Choi Department of Emergency Medicine, Ewha Medical Center, Ewha Womans University School of Medicine, 1071 Anyangcheon-ro, Yangcheon-gu, Seoul 07985, Korea

E-mail: like-lemontea@hanmail.net

\section{Capsule}

Summary

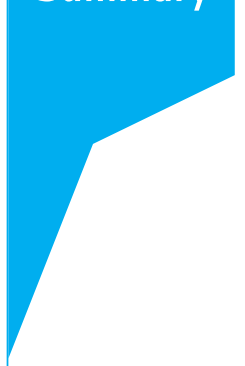

What is already known

Epigastric pain is a common symptom in the emergency department; most cases are not fatal. But epigastric pain can be the only symptom of isolated spontaneous gastric artery dissection.

\section{What is new in the current study}

Epigastric pain can be the only symptom of isolated spontaneous gastric artery dissection. Although it is extremely rare, patients presenting with epigastric pain with risk of vasculopathies should be considered for the possible isolated spontaneous gastric artery dissection. Early recognition and definite therapy lead to favorable outcome.

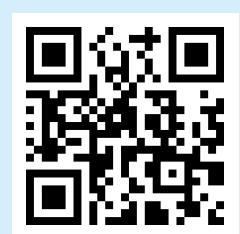

How to cite this article:

Lim JY, Choi YH, Lee SH. Unusual presentation and treatment of isolated spontaneous gastric artery dissection. Clin Exp Emerg Med 2016;3(2):112-115.

This is an Open Access article distributed under the terms of the Creative Commons Attribution Non-Commercial License (http:// creativecommons.org/licenses/by-nc/4.0/). 


\section{INTRODUCTION}

Epigastric pain is a common symptom in the emergency department (ED) and is generally not fatal. However, it can be the only symptom of isolated spontaneous gastric artery dissection. Here, we report the case of a 51-year-old woman who initially presented to the ED with epigastric pain only and was subsequently diagnosed with isolated spontaneous gastric artery dissection and hepatic artery aneurysm.

\section{CASE REPORT}

A 51-year-old woman presented to the ED with epigastric abdominal pain for 2 days. She had been diagnosed with neurofibromatosis 10 years earlier but was not worked up. Two days before admission, she consumed spicy food and subsequently developed a burning, sharp, intermittent, colicky pain with nausea. She denied vomiting, diarrhea, fever, sweating, dyspnea, or any other symptoms including back and chest pain. Her vital signs were as follows: blood pressure, 125/70 mmHg; pulse rate, 80 beats/min; respiratory rate, 20 breaths $/ \mathrm{min}$; and body temperature, $36.0^{\circ} \mathrm{C}$. Physical examination revealed a soft and flat abdomen as well as tenderness without rebound tenderness in the epigastric area; other findings were unremarkable. Her laboratory data were normal, including hemoglobin, platelet count, white blood cell count, and blood chemistry. Abdominal radiography revealed no abnor-

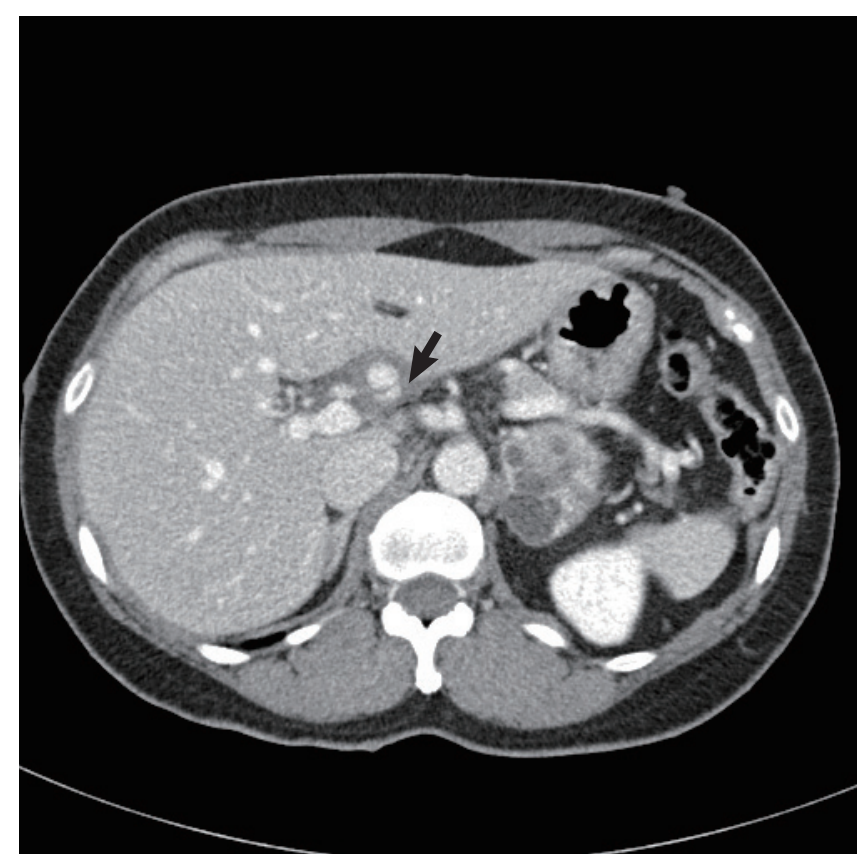

Fig. 1. Abdominopelvic computed tomography showing a left hepatic arterial pseudo-aneurysm with acute hematoma by rupture (arrow). mal bowel gas pattern, abnormal calcific density in the abdominopelvic cavity, or organomegaly. Electrocardiography showed normal sinus rhythm. A provisional diagnosis of gastritis or gastric spasm was made. Fluid therapy with symptomatic treatment was administered. Three hours later, her symptoms (i.e., burning, colicky pain, and epigastric discomfort) improved, and she was discharged with oral medications. Two hours later, she returned to the ED with burning, sharp, persistent epigastric pain; rebound tenderness in the epigastric area with a newly developed Murphy's sign. Vital signs at her second visit were as follows: blood pressure, 110/60 $\mathrm{mmHg}$; pulse rate, 95 beats/min; respiratory rate, 23 breaths $/ \mathrm{min}$; and body temperature, $36.7^{\circ} \mathrm{C}$. Enhanced abdominopelvic computed tomography was performed to exclude acute cholecystitis and showed a left hepatic arterial pseudoaneurysm with an acute hematoma due to its rupture (Fig. 1). Selective celiac angiography revealed a left hepatic arterial aneurysm and right gastric artery dissection (Fig. 2). The left hepatic arterial aneurysm and right gastric artery dissection were embolized. The patient was subsequently managed in the cardiovascular department and discharged without complications 10 days later.

\section{DISCUSSION}

Visceral artery aneurysms are rare. They mainly involve the splenic artery (60\%); other less-frequent visceral sites include the superior mesenteric artery (5.5\%), celiac trunk (4\%), left gastric ar-

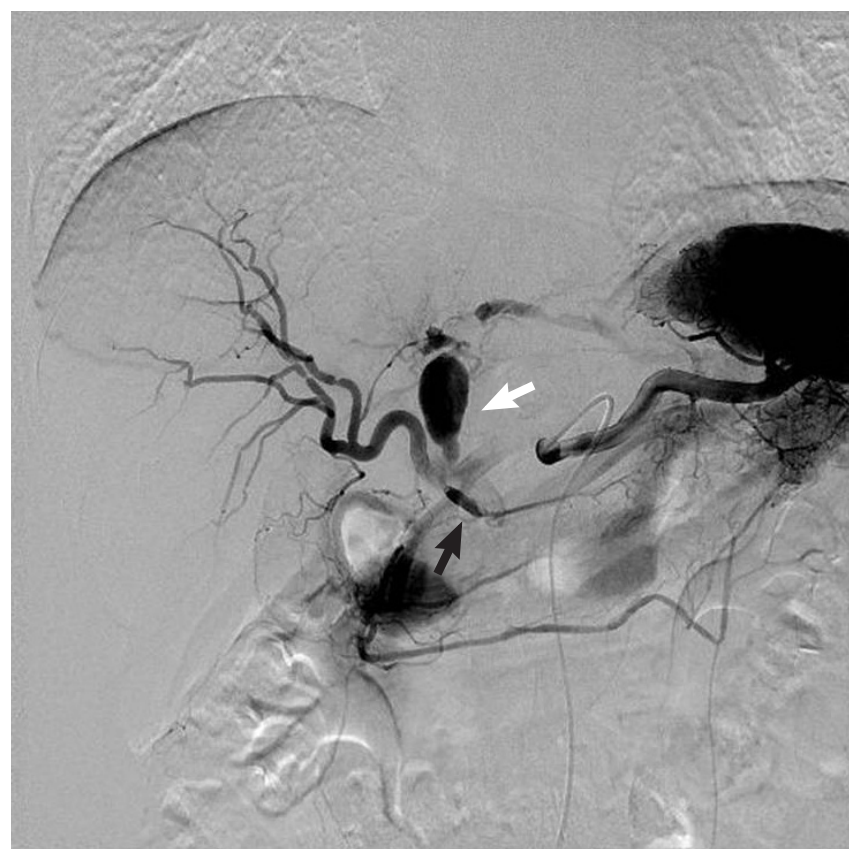

Fig. 2. Angiography showing a left hepatic arterial aneurysm (white arrow) and right gastric artery dissection (black arrow). 
tery (4\%), and gastroepiploic artery (4\%). ${ }^{1,2}$ Visceral artery dissection usually occurs during the extension of aortic dissection. In contrast, spontaneous isolated visceral artery dissection without associated aortic dissection is uncommon. The superior mesenteric artery is the most frequent site of dissection in visceral arteries, and celiac artery dissection is rare. Isolated spontaneous gastric artery dissection is rare, although there are reports of isolated spontaneous celiac artery dissection. Spontaneous visceral artery dissection is five times more common in men than in women, and the average age of patients is approximately 55 years. ${ }^{3}$ The risk factors are hypertension, smoking, connective tissue diseases, atherosclerosis, abdominal trauma, medial degeneration, fibrodysplasia, and vasculitis. ${ }^{4-8}$ Although the clinical presentation varies, almost all cases of celiac artery dissection are characterized by abdominal pain mainly located in the epigastric region accompanied with nausea, vomiting, or diarrhea. ${ }^{7-9}$

Epigastric pain is a common symptom in the ED and is generally not fatal except in acute myocardial infarction, aortic dissection, and abdominal aneurysm rupture. Our patient had hepatic artery aneurysm with right gastric artery dissection. Consequently, she complained only of epigastric pain at the first visit. Given its low incidence and unpredictable natural history, hepatic artery aneurysm is rarely considered in the diagnosis of acute abdominal pain. Depending on the location of the arterial dissecting lesion, jaundice associated with the hepatic artery dissection and pancreatitis with elevated serum amylase levels associated with the dissection of branches of pancreatic artery may be observed. ${ }^{10}$

The diagnosis of artery dissection largely depends on radiological findings. ${ }^{11,12}$ Computed tomography is the best diagnostic modality for this purpose; in addition to being fast and accurate, it provides the most information for making therapeutic decisions. On angiography, artery dissection is characterized by a double lumen, string sign, intraluminal defects, intimal flaps, tapered occlusions, short segmental narrowing, irregular stenosis, and aneurysms."

The disease courses of visceral artery dissection include limited progression with thrombosis of the false lumen, progression of dissection, narrowing or obliteration of the true lumen due to expansion of the false lumen, and rupture. ${ }^{6,13}$ Therefore, management aims to limit the progression of the dissecting hematoma, control hypertension, and prevent thromboembolic complications in order to prevent the expansion of the false lumen, which may cause malperfusion, aneurysmal dilatation, or rupture.

There is currently no optimal treatment strategy for visceral artery dissection. Recommended treatments include open surgery, endovascular treatment, anticoagulation, and conservative treatment. Drug treatments include anticoagulants, anti-inflammatory drugs, steroids, and secondary antiplatelet therapy, which are advisable in patients with stenotic lesions to prevent thrombosis. ${ }^{3,14}$ Endovascular or surgical treatment for revascularization should be considered in patients with persistent abdominal pain, bowel ischemia, necrosis, progression of the dissection, and arterial rupture. ${ }^{5,6}$ Open surgery was the gold standard for a long time. However, endovascular treatment with a stent or embolization is an attractive option for patients at high surgical risk.

Epigastric pain can be the only symptom of isolated spontaneous gastric artery dissection. Although extremely rare, patients presenting with epigastric pain who are at risk of vasculopathy should be considered for isolated spontaneous gastric artery dissection. Early recognition and definitive therapy lead to a favorable outcome.

\section{CONFLICT OF INTEREST}

No potential conflict of interest relevant to this article was reported.

\section{REFERENCES}

1. Messina LM, Shanley CJ. Visceral artery aneurysms. Surg Clin North Am 1997;77:425-42.

2. Untereiner $X$, Kretz $B$, Camin-Kretz $A$, et al. Dissecting aneurysm of the celiac trunk: a case report. Ann Vasc Surg 2014; 28:1037.e1-4.

3. Vaidya S, Dighe M. Spontaneous celiac artery dissection and its management. J Radiol Case Rep 2010;4:30-3.

4. Sakamoto I, Ogawa Y, Sueyoshi E, Fukui K, Murakami T, Uetani $M$. Imaging appearances and management of isolated spontaneous dissection of the superior mesenteric artery. Eur J Radiol 2007;64:103-10.

5. Zhang WW, Killeen JD, Chiriano J, Bianchi C, Teruya TH, AbouZamzam AM. Management of symptomatic spontaneous isolated visceral artery dissection: is emergent intervention mandatory? Ann Vasc Surg 2009;23:90-4.

6. Subhas G, Gupta A, Nawalany M, Oppat WF. Spontaneous isolated superior mesenteric artery dissection: a case report and literature review with management algorithm. Ann Vasc Surg 2009;23:788-98.

7. Ahn S, Sohn CH, Seo DW, Lim KS, Kim W. Diagnosis of spontaneous isolated superior mesenteric artery dissection in the emergency department: a case series. J Korean Soc Emerg Med 2010;21:615-21.

8. Kang TL, Teich DL, McGillicuddy DC. Isolated, spontaneous superior mesenteric and celiac artery dissection: case report and 
review of literature. J Emerg Med 2011;40:e21-5.

9. Park YJ, Park KB, Kim DI, Do YS, Kim DK, Kim YW. Natural history of spontaneous isolated superior mesenteric artery dissection derived from follow-up after conservative treatment. J Vasc Surg 2011;54:1727-33.

10. Chaillou P, Moussu P, Noel SF, et al. Spontaneous dissection of the celiac artery. Ann Vasc Surg 1997;11:413-5.

11. Matsuo R, Ohta Y, Ohya $Y$, et al. Isolated dissection of the celiac artery: a case report. Angiology 2000;51:603-7.
12. Wang HC, Chen JH, Hsiao CC, Jeng CM, Chen WL. Spontaneous dissection of the celiac artery: a case report and literature review. Am J Emerg Med 2013;31:1000.e3-5.

13. Min SI, Yoon KC, Min SK, et al. Current strategy for the treatment of symptomatic spontaneous isolated dissection of superior mesenteric artery. J Vasc Surg 2011;54:461-6.

14. D'Ambrosio N, Friedman B, Siegel D, Katz D, Newatia A, Hines J. Spontaneous isolated dissection of the celiac artery: $\mathrm{CT}$ findings in adults. AJR Am J Roentgenol 2007;188:W506-11. 